\title{
EMPATÍA, ESTILOS DE AFRONTAMIENTO Y ACTITUDES HACIA EL CONSUMO COMO FACTORES DE PROTECCIÓN DEL CONSUMO INTENSIVO DE ALCOHOL Y EL POLICONSUMO EN LA ADOLESCENCIA
}

\author{
Sara González-Yubero, Raquel Palomera Martín y Susana Lázaro-Visa \\ Universidad de Cantabria (España)
}

\begin{abstract}
Resumen
Desde el enfoque del desarrollo positivo adolescente cobra especial importancia la promoción de recursos personales de protección frente a algunos riesgos como el consumo de drogas. El objetivo de este estudio fue analizar la influencia de la empatía, los estilos de afrontamiento y las actitudes hacia el consumo respecto al uso intensivo de alcohol y el policonsumo en 799 estudiantes. Los resultados del análisis de regresión revelaron que el afrontamiento improductivo predijo un mayor consumo intensivo de alcohol, mientras que la actitud de rechazo ante el ofrecimiento de alcohol y la actitud de admiración hacia personas no usuarias de drogas institucionalizadas se asociaron con un menor hábito de consumo. Con relación al policonsumo, tanto la empatía afectiva, como el afrontamiento activo, la actitud contraria y de rechazo al consumo de drogas, así como la actitud de admiración hacia personas no usuarias de drogas institucionalizadas predijeron un menor uso simultaneo de alcohol y cannabis. Estos hallazgos aportan nuevas evidencias que podrían ser de utilidad para orientar intervenciones promotoras de la salud a edades tempranas.

PALABRAS CLAVE: empatía, estilos de afrontamiento, actitudes, consumo intensivo de alcohol, policonsumo, adolescencia.
\end{abstract}

\begin{abstract}
From a positive youth development perspective, the promotion of personal protective resources against certain risks such as drug consumption is especially relevant. The objective of this study was to analyze the influence of empathy, coping styles, and attitudes related to consumption with respect to binge drinking and polyconsumption in a sample of 799 students. The results of a regression analysis revealed that unproductive coping predicted binge drinking, while attitudes such as turning down a drink and admiration of non-users of institutionalized drugs were associated with a lower consumption habit. As for polyconsumption, affective empathy, active coping, a negative attitude to, and the rejection of drug consumption, as well as the admiration of non-users of
\end{abstract}

Los autores quieren agradecer la financiación recibida a través de la convocatoria del Programa de Personal Investigador en Formación Predoctoral de la Universidad de Cantabria (CVE-2016-11670).

Correspondencia: Sara González Yubero, Universidad de Cantabria, Facultad de Educación, Avda. de los Castros, s/n 39005 Santander (España). E-mail: saragonzalezyubero@gmail.com 
institutionalized drugs, predicted a lower simultaneous use of alcohol and cannabis. These findings offer new evidence that may be useful in guiding interventions to promote healthy habits at early ages.

KEY WORDS: empathy, coping styles, attitudes, binge drinking, polyconsumption, adolescence.

\section{Introducción}

Con base en los últimos datos ofrecidos por los organismos internacionales, el consumo de alcohol y cannabis es uno de los principales problemas de Salud Pública en los adolescentes (Observatorio Europeo de las Drogas y las Toxicomanías, 2019). Según la Encuesta Española sobre Uso de Drogas en Estudiantes de Enseñanzas Secundarias (ESTUDES 2018-2019; Plan Nacional sobre Drogas [PNSD], 2019), la prevalencia de consumo habitual de alcohol y cannabis entre los jóvenes de 14 a 18 años es elevada. Una de las cuestiones que más preocupa es la práctica del botellón (binge drinking), que se caracteriza por la ingesta de alcohol en grandes cantidades, concentradas en cortos espacios de tiempo principalmente durante el fin de semana (PNSD, 2019). Los datos muestran que de entre los adolescentes que consumen, un $32,3 \%$ tomó 5 o más vasos de bebidas alcohólicas en un intervalo aproximado de dos horas en el último mes (PNSD, 2019). La edad media de inicio se sitúa en torno a los 14 años, siendo esta práctica similar entre hombres y mujeres y aumentado progresivamente con la edad. El elevado consumo de alcohol por parte de los menores y la forma en que éste se realiza (bebidas de alta graduación, ingesta de alcohol en breves períodos de tiempo, elevado porcentaje de borracheras), hace que esta sustancia siga siendo una prioridad en las estrategias de prevención (Estrategia Nacional sobre Adicciones, 2017; Golpe, Barreiro, Isorna, Varela y Rial, 2017). A pesar de su peligrosidad, el uso de diversas sustancias psicoactivas en un mismo periodo de tiempo también es una práctica extendida entre los adolescentes (PNSD, 2019). Considerando los últimos doce meses, un $42,8 \%$ de los estudiantes realizó algún tipo de policonsumo, siendo este superior en los varones cuando se trata de sustancias ilegales y aumentando conforme avanza la edad. En este contexto, los datos advierten de un incremento del consumo de cannabis entre aquellos que hicieron binge drinking en los últimos 30 días (40,5\%). Así, la literatura ha señalado la relación entre estas prácticas y numerosos riesgos que pueden afectar el desarrollo madurativo, la salud y el ajuste psicosocial de los adolescentes (Alfonso, HuedoMedina y Espada, 2009; Pérez-Fuentes, Molero y Gázquez, 2019; Riquelme, García y Serra, 2018; Tabares et al., 2019), lo que sitúa en un primer plano la necesidad de reforzar las estrategias preventivas.

Aunque una parte importante del trabajo de prevención ha tenido que ver con el estudio de los factores de riesgo relacionados con el uso de sustancias, son los factores personales de protección los que subyacen en esta nueva aproximación. Desde el enfoque del desarrollo positivo del adolescente se adopta una perspectiva centrada en las fortalezas y se expande el concepto de salud incluyendo como necesarias competencias que, a su vez, incrementan la resistencia frente a los riesgos, como el consumo de drogas (Guerra y Bradshaw, 2008). Entre los factores 
de protección en el ámbito del consumo de sustancias, merecen particular atención los estilos de afrontamiento para hacer frente a las dificultades, las actitudes hacia el uso de sustancias que expresan el modo en que los jóvenes se posicionan ante esta problemática, y finalmente las habilidades empáticas, cuya promoción a edades tempranas podría representar un enfoque preventivo novedoso (Laghi, Bianchi, Pompili, Lonigro y Baiocco, 2019; Massey, Newmark y Wakschlag, 2018). Dado que el inicio e incremento del consumo de drogas comienza a edades tempranas (Golpe et al., 2017; Ruiz-Aranda et al., 2010), la adolescencia es un período importante para estudiar la influencia de estos factores respecto al uso de sustancias.

Los estilos de afrontamiento presentan un papel mediador importante entre las situaciones de estrés, los recursos de los adolescentes para abordarlas y las consecuencias derivadas para su salud física y mental (Alonso-Tapia, Rodríguez-Rey, Garrido-Hernansaiz, Ruiz y Nieto, 2019). Cabe distinguir entre un estilo de afrontamiento funcional de aproximación al problema, pudiendo ser este conductual o cognitivo, y un estilo de afrontamiento disfuncional o de evitación que podría favorecer la aparición de problemas psicosociales (Ebata y Moos, 1994). Algunos estudios con adolescentes han encontrado que un menor uso del afrontamiento activo (planificación sobre las formas de resolver el problema, reevaluación cognitiva, búsqueda de apoyo social, etc.), se ha visto relacionado con un incremento de la cantidad y frecuencia de consumo de alcohol y cannabis con efectos persistentes hasta la edad adulta (Lara-Beltrán, 2019; McConnell, Memetovic y Richardson, 2014). Por otra parte, un grupo amplio de investigaciones sugiere que el empleo de un estilo de afrontamiento disfuncional, centrado en evitar o evadirse del problema, negándolo o delegando su solución a factores externos se relaciona con un mayor consumo de estas sustancias en los adolescentes (LaraBeltrán, 2019; Lara-Beltrán, Bermúdez y Pérez-García, 2017; McConnell et al., 2014). Investigaciones recientes advierten de que el consumo elevado de alcohol (González-Yubero, Lázaro-Visa y Palomera, 2020; González-Yubero, Palomera y Lázaro-Visa, 2019) y cannabis (González-Yubero, Palomera, Lázaro-Visa, 2020), podría estar relacionado con el afrontamiento de estados emocionales aversivos a edades tempranas, lo que incrementa la probabilidad de desarrollar problemas de desajuste (Wicki et al., 2017). A pesar de la evidencia previa, son escasas las investigaciones que relacionan los estilos de afrontamiento con el consumo intensivo de alcohol y el policonsumo de alcohol y cannabis en adolescentes, por lo que se hace necesario continuar investigando en esta dirección.

Desde esta aproximación cobra especial importancia el estudio de las actitudes juveniles ante el consumo de sustancias, que se manifiestan a través de las creencias, sentimientos e intenciones de conducta (Moral y Ovejero, 2003). Algunas investigaciones han puesto de relieve como las actitudes favorables hacia el consumo incrementan el abuso de alcohol y cannabis en los adolescentes (Gutiérrez y Romero, 2014; Moreta-Herrera, Ilaja-Verdesoto, Mayorga-Lascano, León-Tamayo y López-Castro, 2018), lo que se asocia a una distorsión de los riesgos derivados de su uso con consecuencias negativas para el ajuste psicosocial (de la Villa, Ovejero, Sirvent, Rodríguez y Pastor, 2009; Oliva, Parra y Sánchez Queija, 2008). En esta línea, mientras que los jóvenes no consumidores muestran actitudes de resistencia a la experimentación y valoran negativamente las consecuencias derivadas de su uso, los 
consumidores reelaboran creencias con base en la necesidad de minimizar sus efectos negativos, valorando en mayor medida la búsqueda de sensaciones, la desinhibición o la euforia (Sirvent, Moral y Rodríguez, 2007). A pesar de que la literatura ha confirmado un vínculo entre las actitudes favorables hacia las drogas y las conductas de consumo (Moreta-Herrera et al., 2018), la idea de que el acceso a sustancias como el alcohol y el cannabis es más difícil para los adolescentes ha recibido cierto apoyo (Cooke et al., 2016) y, en consecuencia, una gran parte de las investigaciones se han llevado a cabo con población adulta y universitaria. Por ello, abordar la problemática descrita a través de estudios que se centren en esta etapa permitiría orientar intervenciones educativas que minimicen los riesgos del consumo intensivo de alcohol y el policonsumo a edades tempranas.

En la actualidad, son escasas las investigaciones que han analizado el papel de la empatía respecto al consumo intensivo de alcohol y el policonsumo en adolescentes. A pesar de ello, la literatura ha demostrado que la empatía se relaciona con un mejor ajuste general en esta etapa (Benita, Levkovitz y Roth, 2017). La empatía es entendida como la capacidad para conectar con los demás y comprende una dimensión emocional (preocupación empática) para generar sentimientos de compasión y preocupación por otros, a la par que una dimensión cognitiva (toma de perspectiva) que hace referencia a la capacidad de representar los pensamientos y motivos de otras personas poniéndose en su lugar (Davis, 1980). A pesar de que son pocos los estudios que analizan el papel protector de las habilidades empáticas sobre el consumo intensivo de alcohol y el policonsumo, algunas investigaciones longitudinales con jóvenes adultos y adolescentes han mostrado que los rasgos asociados con déficits de empatía predicen resultados más severos de consumo de alcohol y drogas (Baskin-Sommers, Waller, Fish y Hyde, 2015; Hyde, Burt, Shaw, Donnellan y Forbes, 2015; Swogger et al., 2016). Así mismo, otros trabajos corroboran la asociación entre una menor empatía y un mayor consumo intensivo de alcohol y cannabis en adolescentes (Laghi et al., 2019; Nguyen, Clark y Belgrave, 2011). Sin embargo, son varios los autores que resaltan la necesidad de explorar por separado las dimensiones de la empatía ya que se ha observado un deterioro del componente afectivo en usuarios dependientes del alcohol y el cannabis (Ferrari, Smeraldi, Bottero y Politi, 2014; Maurage et al., 2011), mientras que otros trabajos confirmaron una mayor dificultad para inferir los pensamientos y emociones de los demás, lo que también implica al sistema de empatía cognitiva (Maurage, Timary, Tecco, Lechantre y Samson, 2015). En esta línea, los recientes resultados de investigación de Laghi et al. (2019), confirmaron la presencia de un patrón disociado de empatía en población adolescente. En su estudio, el deterioro de la empatía afectiva predijo un mayor consumo intensivo de alcohol, mientras que la empatía cognitiva moderó el vínculo entre la resistencia a la prensión del grupo de iguales para beber alcohol y el número de episodios de consumo intensivo de esta sustancia. Estos resultados sugieren que un detrimento de ambos componentes de la empatía podría ser un precursor del abuso de sustancias en adolescentes y no sólo una consecuencia de usuarios con problemas de dependencia. Dado que apenas se encuentran estudios que exploren el efecto de ambos componentes respecto al consumo intensivo de alcohol y el policonsumo de alcohol y cannabis en población 
adolescente, su estudio como variable de protección podría ser especialmente útil a la hora de orientar nuevas acciones preventivas.

Con base en la literatura precedente, y teniendo en cuenta que el uso de sustancias comienza a una edad temprana (Golpe et al., 2017; Ruíz-Aranda et al., 2010), se hace necesario promover investigaciones que ahonden en el papel de los factores personales de riesgo y protección del consumo en la etapa adolescente. Para este trabajo nos planteamos las siguientes hipótesis:

H1: La empatía afectiva y cognitiva, el estilo de afrontamiento activo y de apoyo social, así como las actitudes poco favorables hacia el consumo (concienciación ante los efectos negativos y actitud contraria al consumo de drogas, actitud de resistencia al consumo de drogas, rechazo ante la invitación de tabaco y drogas ilegales, rechazo ante la invitación de alcohol, concienciación ante los efectos negativos y actitud contraria al consumo de drogas institucionalizadas, identificación que se produce entre el consumo de drogas y el hecho de ser mayor, admiración ante los no usuarios de drogas institucionalizadas), correlacionarán de forma negativa y significativa con las variables de consumo intensivo de alcohol y el policonsumo de alcohol y cannabis, al contrario que el estilo de afrontamiento improductivo que correlacionará de forma positiva y significativa con ellas.

H2: Tanto la empatía afectiva y cognitiva, como los estilos de afrontamiento y las actitudes hacia el consumo (concienciación ante los efectos negativos y actitud contraria al consumo de drogas, actitud de resistencia al consumo de drogas, rechazo ante la invitación de tabaco y drogas ilegales, rechazo ante la invitación de alcohol, concienciación ante los efectos negativos y actitud contraria al consumo de drogas institucionalizadas, identificación que se produce entre el consumo de drogas y el hecho de ser mayor, admiración ante los no usuarios de drogas institucionalizadas), tendrán un efecto predictivo sobre los hábitos de consumo intensivo de alcohol y el policonsumo de alcohol y cannabis, que se mantendrá una vez controlado el sexo y la edad de los adolescentes.

\section{Método}

\section{Participantes}

En este estudio participaron un total de diez centros de Educación Secundaria Obligatoria de la comunidad de Cantabria. Se llevó a cabo un muestreo aleatorio estratificado en función de la titularidad del centro escolar (público o privado/concertado) y la localización en entorno rural o urbano, siguiendo la proporción presente en la población de referencia. La muestra final fue de 799 estudiantes con edades comprendidas entre los 12 y los 16 años (12-13 años 38,2\%; 14-16 años $61,8 \% ; M=14,49 ; D T=1,17)$, con una distribución equilibrada por sexo $(51,8 \%$ mujeres y $48,2 \%$ hombres). Un $51.4 \%$ pertenecieron a centros privados/concertados y un $48,6 \%$ a públicos, localizados en el medio urbano (64\%) y rural (36\%). El criterio de exclusión fue encontrarse fuera del rango de edad objeto de estudio 12-16 años ( $n=21$ ), así como no haber rellenado el cuestionario completo al finalizar la segunda sesión $(n=24)$. 


\section{Instrumentos}

a) Cuestionario ad hoc de datos sociodemográficos. Se recogió el sexo y la edad de los participantes.

b) Cuestionario ad hoc de consumo de alcohol. Se realizó una adaptación de dos ítems de ESTUDES (PNSD, 2019): "1) Durante los últimos treinta días, ¿Cuántos días has tomado cinco o más vasos, cañas o copas de bebidas alcohólicas en un intervalo aproximado de dos horas?" y "2) En los últimos 12 meses ¿cuántos días has consumido bebidas alcohólicas y cannabis (hachís o marihuana) sin que hayan pasado más de dos horas entre el consumo de una y otra sustancia?"

c) "COPE-breve" (Brief COPE; Carver, 1997), validado en población española por Crespo y Cruzado (1997). Este autoinforme evalúa las diferentes formas de respuesta al estrés. Consta de 28 ítems agrupados en 14 subescalas y evaluados a través de una escala Likert de cuatro puntos (1= Muy en desacuerdo; 4= Muy de acuerdo). Para este estudio se realizó un análisis factorial cuya solución teórica se ajustó al modelo propuesto por Morán, Landero y González (2010), excepto para la dimensión de Afrontamiento espiritual, que se excluyó por no obtener una saturación factorial significativa. Las dimensiones empleadas en este estudio fueron: 1) Afrontamiento activo del problema, formado por las subescalas de planificación, humor, aceptación, reevaluación cognitiva, autodistracción y actitud de afrontamiento activo; 2) Afrontamiento de apoyo social, con las subescalas de apoyo emocional, apoyo instrumental para pedir consejo o ayuda y expresión de sentimientos; y 3) Afrontamiento improductivo o de evitación, con las subescalas de desconexión conductual, negación, uso de sustancias y autoinculpación. El coeficiente alfa de Cronbach para este estudio fue de $0,72,0,67$ y 0,70 para las tres dimensiones respectivamente.

d) "Autoinforme de consumo de sustancias psicoactivas" (AICA; Santacreu y Froján, 1994). Mide actitudes hacia el consumo de drogas y comprende 30 ítems evaluados mediante una escala Likert de cinco puntos (1= Muy en desacuerdo; $5=$ Muy de acuerdo). Se compone de siete factores: 1) Concienciación ante los efectos negativos y actitud contraria al consumo de drogas (p. ej., "No estaría dispuesto a tomar drogas nunca"); 2) Actitud de resistencia al consumo de drogas (p. ej., "Me alejaría de un sitio en el que se estuviera consumiendo droga"); 3) Rechazo ante la invitación de tabaco y drogas ilegales (p. ej., "Rechazaría una invitación para fumar"); 4) Rechazo ante la invitación de alcohol (p. ej., "Rechazaría una invitación para beber alcohol"); 5) Concienciación ante los efectos negativos y actitud contraria al consumo de drogas institucionalizadas (p. ej., "Me disgusta que se consuma alcohol"); 6) Identificación que se produce entre el consumo de drogas y el hecho de ser mayor (p. ej., "El consumo de alcohol no nos hace mayores"); y 7) Admiración ante los no usuarios de drogas institucionalizadas (p. ej., "Admiro a quien no consume alcohol"). El coeficiente alfa de Cronbach para este estudio fue de 0,78 .

e) "Índice de reactividad interpersonal" (Interpersonal Reactivity Index, IRI; Davis, 1980), validado en población española por Pérez-Albéniz, De Paúl, Etxeberría, Montes y Torres (2003). Mide diferencias individuales en las tendencias 
empáticas a través de 28 ítems evaluados mediante una escala Likert de cuatro puntos (1= Muy en desacuerdo; 4= Muy de acuerdo). Como se ha informado en estudios previos (p. ej., Laghi et al., 2018; Rueda, Fernández-Berrocal y Baron-Cohen, 2015), la dimensión cognitiva y afectiva de la empatía ha sido investigada a través de dos subescalas de este autoinforme: 1) Toma de perspectiva (p. ej., "A veces trato de entender mejor a mis amigos imaginando cómo se ven las cosas desde su punto de vista") y 2) Preocupación empática (p. ej., "A menudo siento ternura y preocupación por las personas menos afortunadas que yo"). En este estudio se obtuvo un alfa de Cronbach de 0,77 y 0,70 respectivamente.

\section{Procedimiento}

Se elaboró un documento informativo dirigido a los centros y a los responsables legales del alumnado para obtener su autorización firmada previa a la participación de los escolares en el estudio. Así mismo, se aseguró el consentimiento informado de los alumnos y su voluntariedad de participación. Un investigador acompañó a los estudiantes en el aula garantizando su anonimato durante el proceso. El tiempo de cumplimentación del instrumento en papel y con bolígrafo fue de dos sesiones de 45 minutos no consecutivas. Los cuestionarios se administraron siguiendo un orden fijo. En primer lugar, se preguntó sobre los hábitos de consumo, posteriormente sobre el modo de afrontamiento y las habilidades empáticas, finalizando con las actitudes hacia el consumo de drogas.

Esta investigación se rige según los principios recogidos en la declaración de Helsinki (World Medical Association, 2013). El desarrollo del plan de investigación del estudio fue aprobado por la Comisión Académica de Estudios de Doctorado de la Universidad de Cantabria.

\section{Análisis de datos}

Para el procesamiento de análisis de datos se empleó el paquete estadístico SPSS Statistics v. 24.0. El estudio se llevó a cabo a través de una metodología de corte cuantitativo y tipo correlacional. En primer lugar, se calcularon los índices de fiabilidad alfa de Cronbach para cada variable estudiada. Del mismo modo, se dicotomizaron las categorías de respuesta de las variables criterio en función de la mediana. A continuación, se realizaron análisis descriptivos de la muestra. Posteriormente, se llevaron a cabo análisis de correlación biserial-puntual de las variables de estudio. En último lugar, se construyeron modelos de regresión logística binaria a partir de las variables predictoras (empatía, estilos de afrontamiento y actitudes hacia el consumo) para observar su asociación respecto a las variables criterio de consumo intensivo alcohol y policonsumo una vez controlado el sexo y la edad de los participantes. El procedimiento empleado fue introducir en los modelos los factores que presentaron correlaciones bivariadas significativas en los análisis previos. Se utilizó el método de pasos hacia atrás extrayendo las variables independientes una a una hasta llegar a un modelo donde todos los factores resultaron significativos (al menos para $p<0,05$ ). Con objeto de sintetizar la 
abundante cantidad de datos, únicamente se presentan los modelos finales que explicaron un mayor porcentaje de la varianza.

\section{Resultados}

Consumo intensivo de alcohol y policonsumo

En la tabla 1 se presentan las cifras de consumo intensivo de alcohol y policonsumo.

Tabla 1

Análisis descriptivo del consumo intensivo de alcohol y el policonsumo

\begin{tabular}{|l|c|c|}
\hline Consumo de alcohol y cannabis & $n$ & $\%$ \\
\hline Consumo intensivo de alcohol & & 32,6 \\
\hline$\leq 5$ días al mes & 105 & 67,4 \\
\hline$\geq 6$ días al mes & 218 & \\
\hline Intensivo alcohol por sexo & 45 & 28,5 \\
\hline Mujer $\leq 5$ días & 113 & 71,5 \\
\hline Mujer $\geq 6$ días & 60 & 36,4 \\
\hline Hombre $\leq 5$ días & 105 & 63,6 \\
\hline Hombre $\geq 6$ días & & \\
\hline Intensivo alcohol por edad & 32 & 38,1 \\
\hline $12-13$ años $\leq 5$ días & 52 & 61,9 \\
\hline $12-13$ años $\geq 6$ días & 73 & 30,0 \\
\hline $14-16$ años $\leq 5$ días & 166 & 70,0 \\
\hline $14-16$ años $\geq 6$ días & & \\
\hline Policonsumo alcohol y cannabis & 46 & 26,0 \\
\hline$<40$ días al año & 138 & 74,0 \\
\hline$\geq 40$ días al año & & \\
\hline Policonsumo por sexo & 19 & 26,8 \\
\hline Mujer $<40$ días & 52 & 73,2 \\
\hline Mujer $\geq 40$ días & 27 & 23,9 \\
\hline Hombre $<40$ días & 86 & 76,1 \\
\hline Hombre $\geq 40$ días & & \\
\hline Policonsumo por edad & 16 & 42,1 \\
\hline $12-13$ años $<40$ días & 22 & 57,9 \\
\hline $12-13$ años $\geq 40$ días & 30 & 20,5 \\
\hline $14-16$ años $<40$ días & 116 & 79,5 \\
\hline $14-16$ años $\geq 40$ días & & \\
\hline
\end{tabular}

En primer lugar, de entre los adolescentes que afirmaron consumir alcohol de manera intensiva $(N=323)$, aproximadamente 7 de cada 10 lo hicieron durante seis días o más en el último mes $(67,4 \%)$. Con relación a las variables sociodemográficas, atendiendo a la muestra de adolescentes consumidores, un 63,6\% de los hombres afirmaron haber consumido alcohol de manera intensiva durante 6 días o más en el último mes, mientras que la prevalencia en las mujeres fue algo superior $(71,5 \%)$. 
Respecto a los grupos de edad, 6 de cada 10 adolescentes de entre 12-13 años afirmaron haber consumido alcohol de manera intensiva durante 6 días o más en el último mes $(61,9 \%)$, siendo este porcentaje superior en el grupo de mayor edad (70\%). En segundo lugar, de entre los policonsumidores $(n=184)$, aproximadamente 7 de cada 10 lo practicaron durante 40 días o más en el último año. Con relación a las variables sociodemográficas se observa que un $73,2 \%$ de las mujeres consumieron alcohol y cannabis al mismo tiempo, mientras que en los hombres la prevalencia fue algo superior $(76,1 \%)$. Respecto al grupo de edad $12-$ 13 años, un 57,9\% lo llevó a cabo durante 40 días o más en el último año, siendo apreciable un incremento del consumo en el grupo de 14-16 años (79,5\%).

\section{Análisis de correlación entre los factores de protección y el consumo}

En la tabla 2 se incluyen la media y la desviación típica de las variables predictoras, así como las correlaciones con las variables criterio de consumo. Se observan correlaciones negativas significativas entre las variables predictoras, el consumo intensivo de alcohol y el policonsumo a excepción del factor de afrontamiento desadaptativo que correlacionó de manera directa y significativa con ellas. El tamaño del efecto de las correlaciones fue bajo (Cohen, 1988).

Tabla 2

Análisis descriptivos y correlacional de los factores de protección personales respecto al consumo intensivo de alcohol y policonsumo

\begin{tabular}{|c|c|c|c|c|c|}
\hline Variable (instrumento) & $M$ & DT & Mín/Máx & Alc. Intens. & Policonsumo \\
\hline 1. Toma de perspectiva (IRI) & 3,90 & 1,1 & $2 / 5$ & $-0,07$ & $-0,16^{*}$ \\
\hline 2. Preocupación empática (IRI) & 3,97 & 0,8 & $2 / 5$ & $-0,11^{*}$ & $-0,21$ ** \\
\hline $\begin{array}{l}\text { 3. Afrontamiento activo } \\
\text { (COPE breve) }\end{array}$ & 32,6 & 6,2 & $10 / 40$ & $-0,16^{*}$ & $-0,23 * *$ \\
\hline $\begin{array}{l}\text { 4. Afrontamiento de apoyo } \\
\text { social (COPE breve) }\end{array}$ & 29,8 & 5,8 & $10 / 40$ & $-0,11$ * & $-0,08$ \\
\hline $\begin{array}{l}\text { 5. Afrontamiento } \\
\text { improductivo (COPE breve) }\end{array}$ & 16,3 & 5,5 & $8 / 40$ & 0,19 ** & $0,15^{*}$ \\
\hline 6. $\quad F 1(A I C A)$ & 3,77 & 1,4 & $1 / 5$ & $-0,18^{* *}$ & $-0,26$ ** \\
\hline 7. $\quad$ F2 (AICA) & 3,00 & 1,4 & $1 / 5$ & $-0,09$ & $-0,19 *$ \\
\hline 8. $\quad$ F3 (AICA) & 3,89 & 1,2 & $1 / 5$ & $-0,16^{*}$ & $-0,28 * *$ \\
\hline 9. $\quad \mathrm{F} 4(\mathrm{AICA})$ & 3,08 & 1,3 & $1 / 5$ & $-0,26 * *$ & $-0,20 *$ \\
\hline 10. F5 (AICA) & 3,61 & 1,3 & $1 / 5$ & $-0,15 * *$ & $-0,19 *$ \\
\hline 11. $\mathrm{F} 6(\mathrm{AICA})$ & 3,98 & 1,3 & $1 / 5$ & $-0,18^{* *}$ & $-0,20 * *$ \\
\hline 12. F7 (AICA) & 3,29 & 1.4 & $1 / 5$ & $-0.27 * *$ & $-0,27 * *$ \\
\hline
\end{tabular}

Notas: Alc. Intens.= alcohol intensivo; $\mid \mathrm{RI}=$ "Índice de reactividad interpersonal"; $\mathrm{AICA}=$ "Autoinforme de consumo de sustancias psicoactivas"; F1= Concienciación ante los efectos negativos y actitud contraria al consumo de drogas; F2= Actitud de resistencia al consumo de drogas; F3= Rechazo ante la invitación de tabaco y drogas ilegales; F4= Rechazo ante la invitación de alcohol; F5= Concienciación ante los efectos negativos y actitud contraria al consumo de drogas institucionalizadas; F6= Identificación que se produce entre el consumo de drogas y el hecho de ser mayor; F7= Admiración ante los no usuarios de drogas institucionalizadas. ${ }^{\star} p<0,05 .{ }^{*} p<0,01$. 
Predicción del consumo en función de los factores de protección personales, el sexo y la edad

Se realizaron análisis de regresión logística binaria para estudiar el efecto de los factores de protección personales respecto al consumo intensivo de alcohol y el policonsumo de alcohol y cannabis una vez controlado el sexo y la edad de los adolescentes. A continuación, se presentan los modelos predictivos finales que explicaron un mayor porcentaje de la varianza. En primer lugar, el modelo obtenido para la variable dependiente "consumo de intensivo alcohol" permitió una estimación correcta del 70,9\% de los casos, cuyos resultados se pueden observar en la tabla 3. Entraron a formar parte de la ecuación las variables independientes de afrontamiento improductivo, rechazo de invitación de alcohol y admiración ante no usuarios de drogas institucionalizadas. El estadístico $R^{2}$ de Nagelkerke estimó un valor de ajuste de 0,176 . En segundo lugar, cuando tomamos como variable criterio el "policonsumo", se obtuvo una estimación correcta del modelo del 82,6\% de los casos, entrando a formar parte de la ecuación la edad, el factor de preocupación empática, afrontamiento activo, concienciación ante los efectos negativos y actitud contraria al consumo de drogas, rechazo de invitación de tabaco y drogas ilegales y finalmente admiración ante no usuarios de drogas institucionalizadas. El estadístico $R^{2}$ de Nagelkerke estimó un valor de ajuste de 0,419.

Tabla 3

Predicción del consumo de alcohol intensivo y policonsumo en función de los factores personales de protección controlado por sexo y edad

\begin{tabular}{|l|c|c|c|c|c|}
\hline \multicolumn{1}{|c|}{ Modelo } & B & E.T. & Wald & OR & IC 95\% \\
\hline Alcohol intensivo & & & & & \\
\hline Afrontamiento improductivo & 0,50 & 0,02 & $6,989^{* *}$ & 1,05 & $1,01 / 1,09$ \\
\hline $\begin{array}{l}\text { Rechazo de invitación de } \\
\text { alcohol }\end{array}$ & $-0,31$ & 0,09 & $11,133^{* *}$ & 0,73 & $0,60 / 0,90$ \\
\hline $\begin{array}{l}\text { Admiración ante no usuarios } \\
\text { de drogas institucionalizadas }\end{array}$ & $-0,29$ & 0,09 & $10,470 * *$ & 0,75 & $0,63 / 0,90$ \\
\hline Constante poblacional & 1,380 & 0,474 & $8,491^{* *}$ & -- & -- \\
\hline Policonsumo & 1,31 & 0,51 & $6,510^{*}$ & 3,69 & $1,35 / 10,06$ \\
\hline Edad & $-0,54$ & 0,22 & $5,839^{*}$ & 0,60 & $0,38 / 0,90$ \\
\hline Preocupación empática & $-0,07$ & 0,03 & $5,922^{*}$ & 0,93 & $0,87 / 0,98$ \\
\hline Afrontamiento activo & $-0,41$ & 0,15 & $7,301^{* *}$ & 0,70 & $0,49 / 0,89$ \\
\hline $\begin{array}{l}\text { Concienciación ante los } \\
\text { efectos negativos y actitud } \\
\text { contraria al consumo de } \\
\text { drogas }\end{array}$ & $-0,38$ & 0,170 & $5,216^{*}$ & 0,70 & $0,49 / 0,95$ \\
\hline $\begin{array}{l}\text { Rechazo de invitación de } \\
\text { tabaco y drogas ilegales }\end{array}$ & $-0,57$ & 0,165 & $11,994^{* *}$ & 0,60 & $0,41 / 0,78$ \\
\hline $\begin{array}{l}\text { Admiración ante no usuarios } \\
\text { de drogas institucionalizadas }\end{array}$ & 6,537 & 1,600 & $16,691^{* *}$ & -- & -- \\
\hline \begin{tabular}{l} 
Constante poblacional \\
\hline
\end{tabular}
\end{tabular}

Nota: ${ }^{*} p<0,01$. 
Como se puede observar, de entre las variables sociodemográficas sólo la edad resultó significativa para la variable dependiente "policonsumo" (tabla 3). A continuación, se estratifican los resultados en función de los dos grupos de edad (12-13 años y 14-16 años) para observar cómo influyen los factores de protección personales sobre el policonsumo en la preadolescencia y adolescencia. En primer lugar, el modelo para el grupo de edad 12-13 años (tabla 4), permitió una estimación correcta del $72,2 \%$ de los casos. Entró a formar parte de la ecuación la variable independiente admiración ante no usuarios de drogas institucionalizadas. El estadístico $R^{2}$ de Nagelkerke estimó un valor de ajuste de 0,240. Así mismo, el modelo creado para el grupo de edad 14-16 años, permitió una estimación correcta del $83,5 \%$ de los casos. Entraron a formar parte de la ecuación las variables independientes: preocupación empática, afrontamiento activo, concienciación ante los efectos negativos, actitud contraria al consumo de drogas y rechazo de invitación de tabaco y drogas ilegales. El estadístico $R^{2}$ de Nagelkerke estimó un valor de ajuste de 0,469 .

Tabla 4

Predicción del policonsumo por grupos de edad

\begin{tabular}{|c|c|c|c|c|c|}
\hline Modelo & B & E.T. & Wald & OR & IC 95\% \\
\hline \multicolumn{6}{|l|}{ Policonsumo 12-13 años } \\
\hline $\begin{array}{l}\text { Admiración ante no usuarios } \\
\text { de drogas institucionalizadas }\end{array}$ & $-0,70$ & 0,30 & $5,843^{*}$ & 0,50 & $0,30 / 0,87$ \\
\hline Constante poblacional & 1,750 & 0,716 & $5,970 *$ & -- & -- \\
\hline \multicolumn{6}{|l|}{ Policonsumo 14-16 años } \\
\hline Preocupación empática & $-0,52$ & 0,26 & $4,100 *$ & 0,59 & $0,36 / 0,98$ \\
\hline Afrontamiento activo & $-0,85$ & 0,04 & $4,830^{*}$ & 0,91 & $0,85 / 0,99$ \\
\hline $\begin{array}{l}\text { Concienciación ante los } \\
\text { efectos negativos y actitud } \\
\text { contraria al consumo de } \\
\text { drogas }\end{array}$ & $-0,47$ & 0,18 & $6,435^{*}$ & 0,63 & $0,44 / 0,90$ \\
\hline $\begin{array}{l}\text { Rechazo de invitación de } \\
\text { tabaco y drogas ilegales }\end{array}$ & $-0,53$ & 0,22 & $5,935^{\star}$ & 0,59 & $0,38 / 0,90$ \\
\hline $\begin{array}{l}\text { Admiración ante no usuarios } \\
\text { de drogas institucionalizadas }\end{array}$ & $-0,54$ & 0,20 & $7,067 * *$ & 0,58 & $0,39 / 0,87$ \\
\hline Constante poblacional & 9,912 & 1,872 & $28,032 * *$ & -- & -- \\
\hline
\end{tabular}

Nota: ${ }^{*} p<0,05 ;{ }^{* *} p<0,01$.

\section{Discusión}

El objetivo principal de esta investigación ha consistido en analizar la influencia de la empatía, los estilos de afrontamiento y las actitudes respecto al consumo intensivo de alcohol y el policonsumo en adolescentes. Así, los hallazgos de este estudio aportan indicios sobre su papel a edades tempranas. Nuestros resultados apuntan que, tanto la empatía afectiva y cognitiva, como el estilo de afrontamiento activo y de apoyo social, así como las actitudes, actuaron como factores de protección de las conductas de consumo, al contrario que el estilo de afrontamiento improductivo, que como se esperaba, actuó como factor de riesgo. Así mismo, cabe 
señalar que el tamaño del efecto de las correlaciones entre las variables predictoras y las variables criterio fue principalmente bajo (Cohen, 1988), al igual que se ha observado en estudios previos sobre el consumo de drogas en jóvenes y adolescentes (Corbin, Farmer y Nolen-Hoekesma, 2013; Laghi et al., 2019; MoretaHerrera et al., 2018). En segundo lugar, una vez se controló el sexo y la edad de los participantes, el afrontamiento improductivo predijo un mayor consumo intensivo de alcohol, mientras que la actitud de rechazo ante el ofrecimiento de alcohol y la actitud de admiración hacia personas no usuarias de drogas institucionalizadas se asociaron a un menor hábito de consumo. Respecto al policonsumo, tanto la empatía afectiva, como el afrontamiento activo, la actitud contraria y de rechazo hacia el consumo de drogas, así como la actitud de admiración hacia personas no usuarias de drogas institucionalizadas, se asociaron a un menor consumo simultaneo de alcohol y cannabis. Estos resultados se mantuvieron para el grupo de 14-16 años en los análisis estratificados, mientras que la actitud de admiración ante no usuarios de drogas institucionalizadas resultó ser un predictor clave para el grupo de menor edad (12-13 años).

Con relación al efecto independiente de la empatía sobre el policonsumo de alcohol y cannabis, cabe señalar que sólo la preocupación empática predijo de forma negativa este hábito en los adolescentes. Los hallazgos de este estudio confirman otras investigaciones en las que se observó un patrón disociado de la empatía respecto al abuso de alcohol, con empatía afectiva deteriorada y empatía cognitiva preservada en los adolescentes (Laghi et al., 2019). Así, nuestros resultados apoyan la idea de que la carencia de empatía emocional está relacionada con el policonsumo en el grupo de mayor edad (14-16), periodo en el que se inicia mayormente el consumo simultaneo de alcohol y cannabis (PNSD, 2019), y no es sólo una consecuencia de la dependencia a estas sustancias. Estos resultados pueden explicarse con base en la confirmación de estudios previos acerca del vínculo entre una mayor empatía e inteligencia emocional en adolescentes que muestran un mejor ajuste psicosocial (Fernández-Berrocal, Ruiz-Aranda, Salguero, Palomera y Extremera, 2018; Méndez-Giménez, Cecchini y García-Romero, 2019) y menos conductas relacionadas con el consumo de alcohol (González et al., 2019; González et al., 2020), tabaco (González-Yubero, Lázaro-Visa y Palomera, 2020) y cannabis (González et al., 2020) a edades tempranas. Algunos autores (Mayssel et al., 2018), han señalado que un menor nivel de empatía podría reducir el efecto de la moral social agudizando las conductas inadecuadas y favoreciendo un mayor consumo de sustancias adictivas en los adolescentes que, a medida que van madurando, se vuelven más sensibles a las necesidades y deseos de los demás, lo que podría explicar la presencia de la empatía afectiva como factor protector del policonsumo en el grupo de mayor edad.

Así mismo, cabe resaltar el papel del estilo de afrontamiento activo con estrategias como la planificación, el humor, la autodistracción o la reevaluación cognitiva, cuya función protectora frente al policonsumo parece cobrar especial importancia. La literatura ha demostrado que un estilo de afrontamiento adaptativo y centrado en la solución del problema facilita el control conductual y emocional promoviendo un mayor ajuste psicosocial y bienestar en los adolescentes (Compas, 1987). Algunos autores señalan que la maduración sociocognitiva se produce en 
torno a los quince años y está vinculada a un incremento de las estrategias de afrontamiento (Seiffge-Krenke, 1998). Nuestros datos se alinean con otras investigaciones que sugieren que los adolescentes de mayor edad emplean estilos de afrontamiento activo del problema con mayor frecuencia que los jóvenes, y son más capaces de controlar los estresores a través de un repertorio de respuestas más amplio (Ebata y Moos, 1994), aspecto que podría explicar el papel protector de este estilo de afrontamiento frente al riesgo de policonsumo en el grupo de mayor edad (14-16 años). Por otro lado, el estilo de afrontamiento improductivo, con presencia de estrategias como la desconexión conductual, negación, uso de sustancias y autoinculpación, predijo un mayor consumo intensivo de alcohol en los adolescentes. Estos datos concuerdan con lo hallado en estudios previos (LaraBeltrán, Bermúdez y Pérez-García, 2017; McConnell et al., 2014), en los que se ha observado una asociación entre el empleo de estilos de evitación y un aumento del consumo abusivo de alcohol y otras sustancias en los adolescentes. Así, algunas investigaciones apuntan que el consumo intensivo de alcohol podría ser una vía para regular los estados emocionales desagradables ante la carencia de recursos propios (González-Yubero et al., 2019; González-Yubero et al., 2020), facilitando así conductas desajustadas y de evitación ante los problemas (Wills y Hirky, 1996).

Del mismo modo, este estudio verifica la asociación entre un menor consumo intensivo de alcohol y policonsumo en adolescentes con una mayor tendencia actitudinal de rechazo del uso de sustancias psicoactivas, una mayor concienciación y deseos de evitar los efectos negativos del consumo, así como una mayor admiración hacia iguales no consumidores de drogas institucionalizadas. Cabe señalar que estos resultados se mantuvieron para la variable de policonsumo en el grupo de mayor edad (14-16 años), periodo en el que un mayor porcentaje de adolescentes se inicia al consumo simultaneo de alcohol y cannabis, mientras que la actitud de admiración a usuarios no consumidores de drogas institucionalizadas fue la única que predijo el policonsumo en los más jóvenes (12-13 años). En las primeras etapas de la adolescencia cobra especial relevancia la identificación con el grupo de iguales, lo que podría incrementar la susceptibilidad a su influencia, modificando el perfil del joven consumidor y las distorsiones en la percepción de riesgo ante drogas como el alcohol y el cannabis (de la Villa et al., 2009). Nuestros resultados están en concordancia con otros estudios (Moreta-Herrera et al., 2018), y apoyan la idea de que una disposición menos favorable al consumo de alcohol y drogas ilegales reduce la participación en conductas de riesgo cómo el consumo intensivo de alcohol y el policonsumo a edades tempranas.

Finalmente, cabe señalar que en esta investigación la empatía cognitiva únicamente correlacionó de manera inversa y significativa con la variable de policonsumo. De igual modo, a pesar de que el afrontamiento de apoyo social correlacionó de forma negativa y significativa con la variable de consumo intensivo de alcohol, posteriormente no mostró un poder explicativo elevado al considerarse el resto de los factores de protección. Respecto a las actitudes, se obtuvieron correlaciones negativas y significativas entre estas, el consumo intensivo de alcohol y el policonsumo, aunque los análisis de regresión ayudaron a identificar los factores de mayor relevancia en su predicción. 
Los hallazgos de este estudio amplían la evidencia previa respecto al valor de protección que ejerce la empatía afectiva, el estilo de afrontamiento adaptativo y las actitudes sobre el riesgo de consumo intensivo de alcohol y policonsumo a edades tempranas. Así, nuestros resultados se alinean con las aportaciones de distintos autores que proponen la promoción de recursos y competencias personales que faciliten el bienestar y la resistencia ante situaciones de riesgo como el consumo de drogas durante esta etapa (Guerra y Bradshaw, 2008), variables que también son puestas de relieve desde los modelos teóricos del Desarrollo Positivo Adolescente. En este sentido, se podría concluir que los adolescentes con mayor habilidad empática para generar sentimientos de compasión y preocupación por los demás, con más capacidad para lidiar de manera adaptativa con las experiencias estresantes que se les presentan, y con actitudes menos favorables hacia el consumo de sustancias psicoactivas tienen menos probabilidades de consumir alcohol de forma intensiva y de llevar a cabo policonsumo de drogas como el alcohol y el cannabis.

Conviene señalar que este estudio cuenta con ciertas limitaciones metodológicas. Así, el hecho de haber empleado una muestra de adolescentes exclusivamente de una comunidad autónoma limita la capacidad de generalizar los resultados a nivel estatal. Del mismo modo, los instrumentos empleados fueron autoinformes, por lo que sería de esperar que futuros estudios pudieran replicar nuestros resultados aplicando medidas más objetivas como los tests de rendimiento máximo. Así mismo, nos gustaría señalar que el instrumento Brief Cope ofreció una fiabilidad adecuada para este estudio, y aunque sí se emplea en población adolescente en otros países (Mohammadzadeh, Awang, Ismail y Shahr, 2020; Schneider, King y Delfabbro, 2018; Yuan, Zhang y Li, 2017), no se ha validado en población española para esta etapa, por lo que se hace necesaria más investigación al respecto. Finalmente, es importante referirse a la naturaleza transversal de esta investigación, por lo que futuros trabajos deberían continuar corroborando los resultados encontrados a través de diseños prospectivos que permitan inferir relaciones de causalidad entre las variables estudiadas. A pesar de estas limitaciones, esta investigación aporta información adicional sobre la relación de los factores de protección personales asociados al consumo intensivo de alcohol y el policonsumo en la etapa más temprana de la adolescencia, periodo en el que se inician estos hábitos nocivos. De este modo, aunque los factores de riesgo que se asocian al consumo son numerosos y muchos no pueden ser modificados, las habilidades incluidas en este estudio pueden ser desarrolladas actuando como factores de protección del consumo en los adolescentes. Por todo ello, consideramos que los hallazgos de esta investigación ofrecen apoyo empírico a los programas educativos focalizados en la promoción de recursos personales para la mejora de estas habilidades.

\section{Referencias}

Alfonso, J. P., Huedo-Medina, T. B. y Espada, J. P. (2009). Factores de riesgo predictores del patrón de consumo de drogas durante la adolescencia. Anales de Psicología, 25(2), 330338.

Alonso-Tapia, J., Rodríguez-Rey, R., Garrido-Hernansaiz, H., Ruiz, M. y Nieto, C. (2019). Coping, personality and resilience: prediction of subjective resilience from coping 
strategies and protective personality factors. Behavioral Psychology/Psicología Conductual, 27(3), 375-389.

Baskin-Sommers, A. R., Waller, R., Fish, A. M. y Hyde, L. W. (2015). Callous-unemotional traits trajectories interact with earlier conduct problems and executive control to predict violence and substance use among high risk male adolescents. Journal of abnormal child psychology, 43(8), 1529-1541. doi: 10.1007/s10802-015-0041-8.

Benita, M., Levkovitz, T. y Roth, G. (2017). Integrative emotion regulation predicts adolescents' prosocial behavior through the mediation of empathy. Learning and Instruction, 50, 14-20. doi: 10.1016/j.learninstruc.2016.11.004.

Carver, C. S. (1997). You want to measure coping but your protocol's too long: consider the Brief COPE. International Journal of Behavioral Medicine, 4(1), 92-100.

Cohen, J. (1988). Statistical power analysis for the behavioral sciences ( $2^{\mathrm{a}}$ ed.). Nueva Jersey, NJ: Lawrence Erlbaum.

Compas, B. E. (1987). Coping with stress during childhood and adolescence. Psychological bulletin, 101(3), 393.

Cooke, R., Dahdah, M., Norman, P. y French, D. P. (2016). How well does the theory of planned behaviour predict alcohol consumption? A systematic review and metaanalysis. Health Psychology Review, 10(2), 148-167. doi: 10.1080/17437199.2014.947547.

Corbin, W. R., Farmer, N. M. y Nolen-Hoekesma, S. (2013). Relations among stress, coping strategies, coping motives, alcohol consumption and related problems: a mediated moderation model. Addictive Behaviors, 38(4), 1912-1919. doi: 10.1016/j.addbeh.2012.12.005

Crespo, M. y Cruzado, J. A. (1997). La evaluación del afrontamiento: adaptación española del cuestionario COPE con una muestra de estudiantes universitarios. Análisis y Modificación de Conducta, 23(92), 797-830.

Davis, M. H. (1980). A multidimensional approach to individual differences in empathy. Catalog of Selected Documents in Psychology, 10, 85, 1-17.

de la Villa Moral Jiménez, M., Ovejero Bernal, A., Sirvent Ruiz, C., Rodríguez Díaz, F. J. y Pastor Martin, J. (2009). Efectos diferenciales sobre las actitudes ante la experimentación con alcohol y la percepción de riesgo en adolescentes españoles consumidores de cannabis y alcohol. Salud Mental, 32(2), 125-138.

Ebata, A. T. y Moos, R. H. (1994). Personal, situational and contextual correlates of doping in adolescence. Journal of Research in Adolescence, 4, 99-125.

Fernández-Berrocal, P., Ruiz-Aranda, D., Salguero, J. M., Palomera, R. y Extremera, N. (2018). La relación del Test de Inteligencia Emocional de la Fundación Botín (TIEFBA) con el ajuste personal y escolar de adolescentes españoles. Revista de Psicodidáctica, 23(1), 1 8. doi: 10.1016/j.psicod.2017.07.001.

Ferrari, V., Smeraldi, E., Bottero, G. y Politi, E. (2014). Addiction and empathy: a preliminary analysis. Neurological Sciences, 35(6), 855-859. doi: 10.1007/s10072-013-1611-6.

Golpe, S., Barreiro, C., Isorna, M., Varela, J. y Rial, A. (2017). La práctica del botellón en adolescentes gallegos: prevalencia, implicaciones y variables asociadas. Behavioral Psychology/Psicología Conductual, 25(3), 529-545.

González-Yubero, S., Palomera, R. y Lázaro-Visa, S. (2019). Trait and ability Emotional Intelligence as predictors of alcohol consumption in adolescents. Psicothema, 31(3), 292-297. doi: 10.7334/psicothema2018.315.

González-Yubero, S., Palomera, R. y Lázaro- Visa, S. (2020). Inteligencia emocional rasgo y habilidad como factores asociados al consumo de cannabis en la adolescencia. Adicciones. Avance de la publicación online. doi:10.20882/adicciones.1364 
González-Yubero, S., Lázaro-Visa, S. y Palomera, R. (2021). ¿Qué aporta la inteligencia emocional al estudio de los factores protectores personales del consumo de alcohol en la adolescencia? Psicología Educativa, 27(1), 27-36. doi: org/10.5093/psed2020a13

González-Yubero, S, Lázaro-Visa, S. y Palomera, R. (2020). The protective association of trait and ability emotional intelligence with adolescent tobacco use. International. Journal of Environmental Research and Public Health, 17(18), 6865. doi: 10.3390/ijerph17186865

Guerra, N. G. y Bradshaw, C. P. (2008). Linking the prevention of problems behaviors and positive youth development: core competencies for positive youth development and risk prevention. New Directions for Child and Adolescent Development, 122, 1-17.

Gutiérrez, M. y Romero, I. (2014). Resiliencia, bienestar subjetivo y actitudes de los adolescentes hacia el consumo de drogas. Anales de Psicología, 30(2), 608-619. doi: 10.6018/analesps.30.2.148131.

Hyde, L. W., Burt, S. A., Shaw, D. S., Donnellan, M. B. y Forbes, E. E. (2015). Early starting, aggressive, and/or callous-unemotional? Examining the overlap and predictive utility of antisocial behavior subtypes. Journal of Abnormal Psychology, 124(2), 329. doi: 10.1037/abn0000029

Laghi, F., Bianchi, D., Pompili, S., Lonigro, A. y Baiocco, R. (2019). Cognitive and affective empathy in binge drinking adolescents: does empathy moderate the effect of selfefficacy in resisting peer pressure to drink? Addictive Behaviors, 89, 229-235. doi: 10.1016/j.addbeh.2018.10.015

Lara-Beltrán, M. D. (2019). Afrontamiento, afecto y tabaco en una muestra de adolescentes españoles. Revista de Psicología Clínica con Niños y Adolescentes, 6(2), 9-14. doi: 10.21134/rpcna.2019.06.2.1

Lara, M. D., Bermúdez, J. y Pérez-García, A. M. (2017). Positividad, estilo de afrontamiento y consumo de tabaco y alcohol en la adolescencia. Electronic Journal of Research in Education Psychology, 11(30), 345-366. doi: 10.14204/ejrep.30.13036

Massey, S. H., Newmark, R. L. y Wakschlag, L. S. (2018). Explicating the role of empathic processes in substance use disorders: a conceptual framework and research agenda. Drug and Alcohol Review, 37(3), 316-332. doi: 10.1111/dar.12548

Maurage, F., Timary, P., Tecco, J. M., Lechantre, S. y Samson, D. (2015). Theory of mind difficulties in patients with alcohol dependence: beyond the prefrontal cortex dysfunction hypothesis. Alcoholism: Clinical and Experimental Research, 39(6), 980988. doi: 10.1111/acer.12717

Maurage, P., Grynberg, D., Noèl, X., Joassin, F., Philippot, P., Hanak, C., Verbanck, P., Luminet, O., de Timary, P. y Campanella, S. (2011). Dissociation between affective and cognitive empathy in alcoholism: a specific deficit for the emotional dimension. Alcoholism: Clinical and Experimental Research, 35(9), 1662-1668. doi: 10.1111/ j.1530- 0277.2011.01512.x

McConnell, M. M., Memetovic, J. y Richardson, C. G. (2014). Coping style and substance use intention and behavior patterns in a cohort of BC adolescents. Addictive behaviors, 39(10), 1394-1397. doi: 10.1016/j.addbeh.2014.05.018

Méndez-Giménez, A., Cecchini, J. A. y García-Romero, C. (2019). Profiles of Emotional Intelligence and Their Relationship with Motivational and Well-being Factors in Physical Education. Revista de Psicología Educativa, 26(1), 27-36. doi: 10.5093/psed2019a19

Mohammadzadeh, M., Awang, H., Ismail, S. y Shahr, H. K. (2020). Improving coping mechanisms of Malaysian adolescents living in orphanages through a life skills education program: a multicentre randomized controlled trial. Asian Journal of Psychiatry, 48, 101892. doi: 10.1016/j.ajp.2019.101892

Moral, M.V. y Ovejero, A. (2003). Actitudes ante el consumo de sustancias psicoactivas y mentalidades del usuario en adolescentes de Secundaria. Entemu, 15, 151-175. 
Morán, C., Landero, R. y González, M. T. (2010). COPE-28: un análisis psicométrico de la versión en español del Brief COPE. Universitas Psychologica, 9(2), 543-552

Moreta-Herrera, R., Ilaja-Verdesoto, B., Mayorga-Lascano, M., León-Tamayo, L. y LópezCastro, J. (2018). Actitudes y disposición personal ante el consumo y exposición a sustancias en adolescentes del ecuador. Salud y Drogas, 18(2). 217-226

Nguyen, A. B., Clark, T. T. y Belgrave, F. Z. (2011). Empathy and drug use behaviors among African-American adolescents. Journal of drug education, 41(3), 289-308. doi: 10.2190/DE.41.3.d

Observatorio Europeo de las Drogas y las Toxicomanías (2019). Informe Europeo sobre Drogas 2019: tendencias y novedades, Oficina de Publicaciones de la Unión Europea, Luxemburgo.

Oliva, A., Ríos, M., Antolín, L., Parra, Á., Hernando, Á. y Pertegal, M. Á. (2010). Más allá del déficit: construyendo un modelo de desarrollo positivo adolescente. Infancia y Aprendizaje, 33(2), 223-234. doi: 10.1174/021037010791114562

Pérez-Albéniz, A., De Paúl, J., Etxeberría, J., Montes, M. P. y Torres, E. (2003). Adaptación de Interpersonal Reactivity Index (IRI) al español. Psicothema, 15(2), 267-272

Pérez-Fuentes, M., Molero, M. y Gázquez, J. J. (2019). Expectativas y búsqueda de sensaciones predictores del binge drinking en adolescentes. Anales de Psicología, 35(1), 124-130. doi: 10.6018/analesps.35.1.308511

Plan Nacional sobre Drogas (2019). Encuesta sobre el uso de drogas en enseñanzas secundarias en España (ESTUDES 2018-2019). Madrid: Ministerio de Sanidad, Servicios Sociales e Igualdad.

Plan Nacional sobre Drogas (2017). Estrategia Nacional sobre Drogas 2017-2024. Madrid: Delegación del Gobierno para el Plan Nacional sobre Drogas, Ministerio de Sanidad y Consumo.

Riquelme, M., Garcia, O. F. y Serra, E. (2018). Psychosocial maladjustment in adolescence: parental socialization, self-esteem, and substance use. Anales de Psicología, 34(3), 536. doi: 10.6018/analesps.34.3.315201

Rueda, P., Fernández-Berrocal, P. y Baron-Cohen, S. (2015). Dissociation between cognitive and affective empathy in youth with Asperger syndrome. European Journal of Developmental Psychology, 12(1), 85-98. doi: 10.1080/17405629.2014.950221

Ruiz-Aranda, D., Cabello, R., Salguero, J. M., Castillo, R., Extremera, N. y Fernández-Berrocal, P. (2010). Los adolescentes malagueños ante las drogas: la influencia de la inteligencia emocional. Málaga: GEU.

Santacreu, J. y Froján, M. X. (1994). Evaluación del consumo de drogas. En R. FernándezBallesteros (dir.), Evaluación conductual hoy. Un enfoque para el cambio en psicología clínica y de la salud (pp. 571-612). Madrid: Pirámide.

Schneider, L. A., King, D. L. y Delfabbro, P. H. (2018). Maladaptive coping styles in adolescents with Internet gaming disorder symptoms. International Journal of Mental Health and Addiction, 16(4), 905-916

Seiffge-Krenke, I. (1998). Adolescents' health. A developmental perspective. Nueva Jersey, NJ: Lawrence Erlbaum.

Sirvent, C., Moral, M. V. y Rodríguez, F. J. (2007). Jóvenes y alcohol: conjunto didácticoaudiovisual para educadores, psicólogos, padres y colectivos mediadores. Valencia: Nau Llibres.

Swogger, M. T., Conner, K. R., Caine, E. D., Trabold, N., Parkhurst, M. N., Prothero, L. M. y Maisto, S. A. (2016). A test of core psychopathic traits as a moderator of the efficacy of a brief motivational intervention for substance-using offenders. Journal of Consulting and Clinical psychology, 84(3), 248. doi: 10.1037/ccp0000065. 
Tabares, G., Stiths, A., Nuñez, C., Caballo, V. E., Agudelo Osorio, M. P. y Grisales Aguirre, A. M. (2019). Predictores psicológicos del riesgo suicida en estudiantes universitarios. Behavioral Psychology/Psicología Conductual, 27(3), 391-413.

Wicki, M., Kuntsche, E., Eichenberger, Y., Aasvee, K., Bendtsen, P., Dankulincová Veselská, Z., Demetrovics, Z., Dzielska, A., Farkas, J., Gaspar de Matos, M., Roberts, C., Tynjälä, J., Välimaa, R. y Vieno, A. (2017). Different drinking motives, different adverse consequences? Evidence among adolescents from 10 European countries. Drug and alcohol review, 36(6), 731-741. doi: 10.1111/dar.12572

Wills, T. A. y Hirky, A.E. (1996). Coping and substance abuse: a theoretical model and review of the evidence. En M. Zeidner y N. S. Endler (dirs.), Handbook of coping. Theory, research, applications (pp. 279-302). Nueva York, NY: John Wiley \& Sons.

World Medical Association (2013). World Medical Association Declaration of Helsinki: ethical principles for medical research involving human subjects. JAMA, 310, 2191-2194. doi: 10.1001/jama.2013.281053

Yuan, W., Zhang, L. F. y Li, B. (2017). Adapting the Brief COPE for Chinese adolescents with visual impairments. Journal of Visual Impairment \& Blindness, 111(1), 20-32. doi: $10.1177 / 0145482 \times 1711100103$

RECIBIDO: 30 de junio de 2020

ACEPTADO: 20 de marzo de 2021 